\title{
ERP-Ausbildung in der Wirtschaftsinformatik an der Universität Stuttgart
}

\author{
Systematische Ableitung eines Lehrkonzepts \\ auf Basis von Lehrzielen
}

\author{
Georg Herzwurm, Lars Oliver Mautsch, Sixten Schockert \\ Lehrstubl für ABWL und Wirtschaftsinformatik II (Unternehmenssoftware), \\ Betriebswirtschaftliches Institut, \\ Universität Stuttgart
}

\section{Einleitung und Ausgangssituation}

Die Wirtschaftsinformatik beschäftigt sich mit der Entwicklung und Anwendung von Theorien, Konzepten, Modellen, Methoden und Werkzeugen für die Analyse, Gestaltung und Nutzung von Informationssystemen (Kurbel 2009, S. 21). Enterprise Resource Planning (ERP) Systeme repräsentieren als integrierte Anwendungssysteme zur Unterstützung nahezu sämtlicher betrieblicher Geschäftsprozesse das automatisierte Teilsystem eines umfassenden betrieblichen Informationssystems. ERP-Systeme sind damit ein zentrales Erkenntnis- und Gestaltungsobjekt der Wirtschaftsinformatik (WI). Viele Autoren weisen zudem darauf hin, dass Absolventen bessere Chancen auf dem Arbeitsmarkt haben, wenn sie Erfahrungen mit ERP-Systemen im Studium vorweisen können (Boyle 2007; Johnson et al.. 2004; Rang 2008; Strong et al. 2006). Es steht somit außer Frage, dass die Vermittlung von Kenntnissen zur Entwicklung und Anwendung von ERP-Systemen ein Bestandteil einer fundierten Wirtschaftsinformatik-Ausbildung sein muss. Sie ist als solches Bestandteil der Rahmenempfehlung für die Universitätsausbildung in der WI (Kurbel 2009, S. 26).

Im Rahmen der Studie von Strong et al.. (2006, S. 9ff) wurde anhand von fünf betrachteten Universitäten gezeigt, dass eine erfolgreiche Integration von ERP in die Lehre nicht immer einfach ist und demzufolge eine sorgfältige Planung benötigt. Dabei sollte die Gestaltung eines Lehrkonzepts zur ERP-Ausbildung - neben Randbedingungen wie die Rahmenempfehlungen zur WI-Ausbildung - auch die Bedürfnisse der beteiligten Personengruppen, im Kern Lehrende und Lernende, berücksichtigen. Um dies in systematischer Weise vorzunehmen, wird in diesem Beitrag eine Methode zur Ableitung eines ERP-Curriculums vorgestellt. Das Vor- 
gehen basiert auf der sehr flexibel einsetzbaren, ursprünglich aus dem Qualitätsmanagement kommenden Planungsmethode Quality Function Deployment (QFD, siehe z. B. Cohen 1995, Herzwurm u. a. 2000). Das wesentliche Kennzeichen von QFD ist die konsequente Orientierung an und Umsetzung von Kundenbedürfnissen bzw. Kundenanforderungen. Im Beispiel der Planung eines Curriculums nehmen Lehrziele diese Rolle der Anforderungen an das Lehrkonzept ein. Diese werden mit potentiellen Lerneinheiten eines ERP-Curriculums korreliert. Durch Bewertung der Lehrziele aus verschiedenen Sichten wie z. B. Dozenten, Studierende oder auch potentielle Arbeitgeber lassen sich mittels dieser Korrelationen Schwerpunkte für die Ausgestaltung eines ERP-Curriculums eben aus diesen potentiell unterschiedlichen Perspektiven ableiten. Auf diese Weise können die im Hochschulbereich in aller Regel knappen Ressourcen (Personal, Budget, Räume, Rechner etc.) gezielt zugewiesen werden. Ebenfalls ist es möglich, ein bestehendes Curriculum zu bewerten und Verbesserungspotentiale aufzudecken.

In Kapitel zwei werden zuerst Lehrziele einer ERP-Ausbildung aus den grundsätzlichen Aufgaben der WI abgeleitet. Daraufhin werden in Kapitel drei potentielle Möglichkeiten dargestellt, diesen Herausforderungen für die Lehre in Form von Lerneinheiten zu begegnen. Auf Basis einer Gewichtung der Lehrziele aus Dozentensicht und einer Beurteilung des Abdeckungsgrades von Lehrzielen durch Lerneinheiten wird in Kapitel vier das ERP-Curriculum der Universität Stuttgart bewertet. Exemplarisch wird dabei die Durchführung einer vergleichenden Fallstudie zur erweiterten Auftragsabwicklung zur Umsetzung in verschiedenen ERPSystemen beschrieben. Abschließend erfolgt in Kapitel fünf eine kurze Diskussion des Konzepts und potentieller Erweiterungen zur Planung eines ERP-Curriculums.

\section{Lehrziele einer ERP-Ausbildung in der WI}

Die Lehrziele einer ERP-Ausbildung konstituieren sich in diesem Beitrag aus der (theoretischen) Sicht der Wirtschaftsinformatik d. h. aus dessen Aufgabe, die Analyse, Gestaltung und Nutzung von Informationssystemen zu lehren.

In der Analyse von Informationssystemen werden anfänglich unbekannte Elemente und Beziehungen innerhalb eines Systems und im Verhältnis zu seinem Kontext in einer sukzessiven Annäherung ermittelt. Dabei können organisatorische (z. B. die zu erfüllenden Aufgaben und ihre Aufgabenträger), soziale (z. B. die Beziehungen der Aufgabenträger untereinander) und technische (z. B informationstechnische Infrastruktur) Dimensionen unterschieden werden (Wyssusek 2001, S. 452). Sie hat das Ziel, mittels Ist-Analyse und Anforderungserhebung ein Sollkonzept inkl. grober informationstechnischer Realisierung für das geplante Anwendungssystem zu entwickeln (Stahlknecht und Hasenkamp 2005, S. 226). Für die ERP-Ausbildung resultieren aus diesem Aspekt drei Lehrziele:

Lehrziel 1 (Z1): ERP-System im Zusammenspiel mit der Ist-Situation im Unternehmen beurteilen und Handlungsempfehlungen aussprechen können. 
Ohne genaue Untersuchung der Ausgangssituation, d. h. insbesondere der zu unterstützenden Geschäftsprozesse im Unternehmen, ist sowohl eine Empfehlung für oder gegen ein (spezielles) ERP-System als auch eine Einschätzung des Veränderungsbedarfs von bestehender Organisation bzw. Softwareunterstützung und potentiellem Standard-ERP-System nicht möglich.

Lehrziel 2 (Z2): Anpassungsbedarf von ERP-Systemen und Unternehmensabläufen identifizieren und bescbreiben können.

Insbesondere fachliche Anpassungen sowohl softwareseitig als auch in bestehenden organisatorischen, abteilungsübergreifenden Geschäftsprozessen sind auf Basis von Analysetätigkeiten zu identifizieren und möglichst integriert, zueinander konsistent zu beschreiben.

Lehriel 3 (Z3): Integrationsbedarfe zwischen Anwendungssystemen identifizieren und beschreiben können.

ERP-Systeme als Teil von Informationssystemen sind immer in einen Kontext eingebettet, der nicht nur aus den organisatorischen Abläufen und Randbedingungen eines Unternehmens besteht, sondern auch aus anderen Anwendungssystemen innerhalb und außerhalb des Unternehmens. Mit diesen muss das ERP-System zusammen arbeiten $\mathrm{d}$. $\mathrm{h}$. die durch diese Integrationsnotwendigkeit induzierten Veränderungsbedarfe sowohl des ERP-Systems als auch angrenzender Systeme sind zu identifizieren und zu beschreiben.

Anpassungs- und Integrationsbedarfe münden in einem Sollkonzept als Ergebnis der Analysetätigkeiten. Die Gestaltung von Informationssystemen setzt auf diesem Sollkonzept auf und hat das Ziel entlang der drei grundsätzlichen Analysedimensionen die Implementierung des ERP-Systems im Unternehmen zu leisten. Demzufolge gehört dazu nicht nur die informationstechnische Umsetzung in SW und HW, sondern auch eine organisatorische Einbettung des ERP-Systems in die Unternehmensabläufe, welche wiederum untrennbar verbunden ist mit der Überwindung und Gestaltung von sozialen Aspekten. Für die ERP-Ausbildung ergeben sich daraus folgende Lehrziele:

Lehriziel 4 (Z4): Anpassen des ERP-Systems an die Belange des Unternehmens vornehmen können.

Hierunter fallen die konkrete Durchführung der als notwendig erkannten Anpassungen im ERP-System durch Parametrisierung, Modifikation oder/und Erweiterung bis hin zur Neu- bzw. Umprogrammierung der von den ERP-Herstellern ausgelieferten Standardversionen einschließlich notwendiger Einrichtungsaktivitäten.

Lebriziel 5 (Z5): Organisatorische Implementierung des ERP-Systems meistern können. Selbst (vermeintlich) unternehmensspezifisch maßgeschneiderte ERP-Systeme sind ohne entsprechende auf das Unternehmen abgestimmte technische, organisatorische und personelle Einführungsaktivitäten nicht produktiv einsetzbar.

Die Nutzung von Informationssystemen bezieht sich dann auf die Unterstützung aller Personen(gruppen), die von dem Informationssystem betroffen sind (auch Stakeholder genannt, Pohl und Rupp 2009, S. 12). Dabei lässt sich in einer einfa- 
chen Unterscheidung die fachliche Nutzung durch (direkte) Anwender bzw. die Interaktion/Integration mit anderen Anwendungssystemen von der informationstechnischen Administration durch Techniker trennen. Daraus ergeben sich für die ERP-Ausbildung unmittelbar folgende beiden Lehrziele:

Lehrziel 6 (Z6): Mit ERP-System fachlich interagieren können.

Lehrziel 7 (Z7): ERP-System technisch administrieren können.

\section{Lerneinheiten in einem ERP-Curriculum}

Im Folgenden werden potentielle Lerneinheiten eines ERP-Curriculums beschrieben, die an der Universität Stuttgart bei der Planung in Betracht gezogen wurden. Sie sind aus gängiger ERP- bzw. WI-Fachliteratur (wie z. B. Hesseler und Görtz 2008, Stahlknecht und Hasenkamp 2005) abgeleitet. Auf dezidierte Literaturhinweise wird deswegen weitgehend (und aus Platzgründen) verzichtet.

LE1 - Prozessanalyse: Im Rahmen der Lerneinheit Prozessanalyse sollen die Studenten in die Lage versetzt werden, (Geschäfts-)Prozesse systematisch zu analysieren sowie übersichtlich und konsistent zu dokumentieren. Die Identifikation von Schwachstellen und Verbesserungspotenzialen z. B. an Schnittstellen zwischen Organisationseinheiten steht im Vordergrund.

LE2 - Prozessmodellierung: Die natürlichsprachige Dokumentation von Geschäftsprozessen stößt gerade bei komplexen, in einen vielschichtigen Kontext eingebetteten Systemen - wie ERP-Systemen - an ihre Grenzen. Zur konsistenten und eindeutigen Beschreibung von Ist- wie Soll-Prozessen sind modellbasierte Dokumentationstechniken wie z. B. die UML (Unified Modelling Language) und ARIS (Architektur integrierter Informationssysteme) notwendig.

LE3 - Erstellung eines Fachkonzepts: Ein IT-Fachkonzept beschreibt aus fachlicher Sicht die Leistungsmerkmale, die der Auftraggeber an eine Software stellt. Die Studierenden sollen lernen, relevante Anforderungen an eine ERP-Software (modellbasiert und natürlichsprachig) zu formulieren und ein in sich stimmiges, die verschiedenen Perspektiven wie die Daten-, Funktions- und Ablaufsicht integrierendes Fachkonzept erstellen zu können.

LE4 - Einrichtung von Mandanten in einer ERP-Software: Ein Mandant gilt als datentechnisch abgeschlossene Einheit innerhalb eines ERP-Systems für ein spezielles Unternehmen. In dieser Lerneinheit werden dazu die grundlegenden Einstellungen auf Basis eines „leeren" Standardmandanten d. h. eines Mandanten ohne Daten gelehrt. Dazu gehören insb. Einrichtungsaktivitäten der Finanzbuchhaltung wie die Festlegung von Kontenplan, Buchungsmatrizen, Analysedimensionen etc. als auch die Problematik des Imports bereits existierender Daten aus Alt-Systemen.

LE5 - Parametrisierung/Customizing ERP-Software: Im Rahmen dieser Lerneinheit werden die Möglichkeiten des Customizing eines ERP-Systems, d. h. alle Einstellungen und Anpassungen, die ohne Programmierung mit Hilfe von Parametern vorgenommen werden können, dargestellt und an den Systemen nachvollzogen. 
Die Studierenden sollen nach dieser Lerneinheit in der Lage sein, eine integrierte betriebliche Standardsoftware ohne (wesentliche) Neu- und Erweiterungsprogrammierung an die Aufbau- und Ablauforganisation eines individuellen Unternehmens anzupassen.

LE6 - Eigenentwicklung von Modulen/Modifikation von ERP-Software: Im Gegensatz zur Lerneinheit LE5 beschäftigt sich diese Lerneinheit mit der individuellen Entwicklung von Modulen durch Programmierung/Coding. Darüber hinaus finden eine Diskussion der Vor- und Nachteile von nicht standard-konformen Eigenentwicklungen und ein Vergleich mit dem Customizing statt.

LE7 - Bearbeitung typischer Geschäfsvorfälle entlang der Supply Chain: Die Intention dieser Lerneinheit ist es, den praktischen Einsatz von ERP-Systemen in Unternehmen entlang der Supply Chain darzustellen. Den Studierenden sollen ein Gefühl für die Komplexität einer integrierten, betriebswirtschaftlichen Standardsoftware und die Sichtweisen der unterschiedlichen mit dem System interagierenden Benutzergruppen vermittelt werden.

LE8 - Wissensvermittlung/Schulung: Die Studierenden sollen durch diese Lerneinheit in die Lage versetzt werden, erlerntes Wissen über eine ERP-Software anderen Anwendern weiterzuvermitteln und damit die Rolle eines Multiplikators einzunehmen. Eine mögliche Lehrform ist, dass jeder Teilnehmer ein Themengebiet vorbereitet und dieses anderen Studierenden vermittelt.

LE9 - Auswablprozesse für ERP-Systeme: Durch die Teilnahme an dieser Lerneinheit werden die Studierenden in die Lage versetzt, systematische und zielorientierte Auswahlprozesse für ERP-Systeme auf Basis von (Kunden-) Anforderungen praktisch durchzuführen. Hierbei lernen sie u. a. unterschiedliche Vorgehensmodelle zur Auswahl kennen und zu beurteilen, eine Zustandsanalyse vorzunehmen und mögliche Alternativen zu identifizieren.

LE10 - Kriterien zur Beurteilung von ERP-Systemen: Die Inhalte von LE10 sollen die Studierenden in die Lage versetzen, einen sinnvollen Kriterienkatalog für die Beurteilung und Auswahl von ERP-Systemen zu erstellen und gängige Fehler bei der Erstellung eines Kriterienkataloges (z. B. zu umfangreiche und standardisierte Kriterienkataloge) zu vermeiden.

LE11 - Einfübrung von ERP-Software in Unternehmen: Diese Lerneinheit behandelt die Einführung einer ERP-Software in Unternehmen als bedeutendes Projekt für das Unternehmen. Die Studierenden lernen u. a. verschiedene Einführungsstrategien zu beurteilen, Besonderheiten des Projektmanagements bei ERPEinführungsprojekten und Vorgehensmodelle kennen sowie gängige Fehler zu vermeiden.

LE12 - Geschäftsmodelle von ERP-Herstellern: Es werden die Geschäftsmodelle ausgewählter ERP-Hersteller vorgestellt und verglichen. Dies versetzt die Studierenden in die Lage, den Markt für ERP-Software zu beurteilen sowie darauf aufbauend einen oder mehrere zu einem Unternehmen passende Hersteller zu identifizieren und in Kombination mit dem Wissen aus LE9 auszuwählen. 
LE13 - Software-Architekturen von ERP-Systemen: Diese Lerneinheit behandelt verbreitete (technische) Architekturen von ERP-Systemen im Allgemeinen, wie z. B. Client-Server-Architekturen oder Service-orientierte Architekturen aber auch spezifischen Modularchitekturen oder Schichtenmodelle einzelner und verbundener ERP-Systeme.

LE14 - Erfolgsfaktoren von ERP-Projekten in der Praxis: Die Studierenden lernen in dieser Lerneinheit Erfolgsfaktoren (aber auch Misserfolgsfaktoren) von ERPProjekten in der Praxis kennen. Dadurch sollen sie in die Lage versetzt werden, gängige Fehler zu vermeiden und ERP-Projekte erfolgreich zu begleiten bzw. abzuschließen.

LE15 - Bedienung: Ziel dieser Lerneinheit ist es, die Studierenden in die Lage zu versetzen, die grundlegenden Bedienkonzepte (inkl. Besonderheiten) der ERPSoftware zu verstehen und die Systeme eigenständig bedienen zu können.

\section{Bewertung und Umsetzung des ERP-Curriculums}

Die Lehrziele und Lerneinheiten eines ERP-Lehrkonzepts aus den beiden vorangegangen Kapiteln sind nun miteinander in Beziehung zu setzen. Allerdings bringt ein einfaches Korrelieren mit dem Ergebnis, „die Lerneinheit Y unterstützt Lehrziel X“ keine wirkliche Entscheidungsunterstützung hinsichtlich des ERPCurriculums. Zum einen sind die Lehrziele untereinander in aller Regel nicht gleich bedeutend für das zu entwickelnde Curriculum. Zum anderen unterstützen die Lerneinheiten auch nicht alle mit gleicher Stärke die Erfüllung der jeweiligen Lehrziele. Beide Aspekte werden in systematischer Weise von Quality Function Deployment (QFD) adressiert und in den Kap. 4.1 und 4.2 dargestellt. Das an der Universität Stuttgart auf Basis der Planungsergebnisse etablierte ERP-Curriculum wird dann in Kap. 4.3 beschrieben.

\subsection{Bewertung der Lehrziele durch die Dozenten}

Innerhalb QFD gibt es mehrere Möglichkeiten, Anforderungen d. h. die Lehrziele zu priorisieren (z. B. Herzwurm u. a. 2000, S. 59, 67). Es lassen sich zumindest einfache Punktbewertungen, absolute Gewichtungen auf Ordinalskalen von z. B. 1 bis 10 und Bewertungen mit dem Ziel, relative Werte zu erhalten, unterscheiden. Letzteres lässt sich bei insgesamt sieben Lehrzielen noch sehr anschaulich mittels eines paarweisen Vergleichs erreichen. Um dabei auch wirklich eine stärkere Differenzierung in der Zielgewichtung zu erhalten, werden in QFD mindestens fünf Kategorien der Vergleichsbeziehung genutzt mit einer dementsprechenden Zuordnung von Zahlenwerten zu den einzelnen Kategorien, i. d. R. im Intervall $[1 / 9,9]$, wobei 1 für ,gleichwichtig" steht.

Nachfolgende Tabelle 1 zeigt die Bewertung der Lehrziele eines ERPCurriculums mittels paarweisen Vergleichs durch die Dozenten der Universität 
Stuttgart. Unterhalb der mit Einsen gefüllten Diagonalen einer Matrix werden die Werte gemäß des Verhältnisses der „Zeilen“ zu den „Spalten“ eingetragen (in der Tabelle hell dargestellt), oberhalb der Diagonalen die reziproken Werte für die entgegengesetzten Beziehungen. Nach der Normalisierung der Spalten erfolgt die Gewichtung durch zeilenweises Summieren der Matrixwerte und Division durch die absolute Anzahl der verglichenen Items.

Tabelle 1: Bewertung der Lehrziele mittels paarweisen Vergleichs

\begin{tabular}{|c|c|c|c|c|c|c|c|c|}
\hline \multirow[t]{2}{*}{$\begin{array}{l}\text { Legende: } \\
\text { Kriterium [Zeile ist mir im } \\
\text { Vergleich zu Kriterium [Spalte] } \\
1 / 9 \text { : viel weniger wichtig } \\
1 / 3: \text { w eniger wichtig } \\
1: \text { : genauso wichtig } \\
3: \text { wichtiger } \\
9 \text { : viel wichtiger } \\
\qquad \text { Z1: Ist-Situation beurteilen }\end{array}$} & 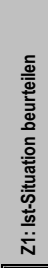 & 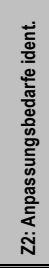 & 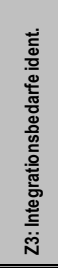 & 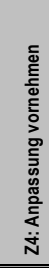 & 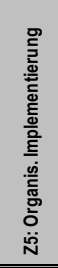 & 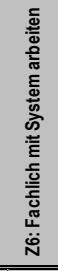 & 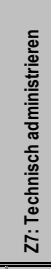 & 9 \\
\hline & 1 & 1 & 3 & 3 & 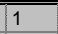 & 1 & 9 & $22,3 \%$ \\
\hline Z2: Anpassungsbedarfe ident. & 1 & 1 & 1 & 3 & 3 & 1 & 9 & $22,1 \%$ \\
\hline Z3: Integrationsbedarfe ident. & $1 / 3$ & 1 & 1 & 3 & 1 & 3 & 9 & $18,8 \%$ \\
\hline Z4: Anpassung vornehmen & $1 / 3$ & $1 / 3$ & $1 / 3$ & 1 & $1 / 3$ & 1 & 1 & $6,1 \%$ \\
\hline Z5: Organis. Implementierung & 1 & $1 / 3$ & 1 & 3 & 1 & 3 & 3 & $16,4 \%$ \\
\hline Z6: Fachlich mit System arbeiten & 1 & 1 & $1 / 3$ & 1 & $1 / 3$ & 1 & 3 & $10,9 \%$ \\
\hline Z7: Technisch administrieren & $1 / 9$ & $1 / 9$ & $1 / 9$ & 1 & $1 / 3$ & $1 / 3$ & 1 & $3,4 \%$ \\
\hline 9 & $47 / 9$ & $47 / 9$ & $67 / 9$ & 15 & 7 & & & $832 / 3$ \\
\hline
\end{tabular}

Die Bewertung der Lehrziele durch die Dozenten zeigt eine Dreiteilung. Die Lehrziele Z1 („Ist-Situation beurteilen“) und Z2 („Anpassungsbedarf identifizieren“), beide begründet in Analysetätigkeiten bestehender und zukünftiger Anwendungssysteme, sind die Bedeutendsten. Dies reflektiert die Ausrichtung der ERPAusbildung an der Universität Stuttgart sowohl auf Studierende der Wirtschaftsinformatik als auch der Betriebswirtschaftslehre. Etwas weniger wichtig sind die Lehrziele Z3 („Integrationsbedarf identifizieren“) und Z5 („Organisatorische Implementierung meistern"), beides in klassischer Weise Arbeitsgebiete von Wirtschaftsinformatikern an der Schnittstelle von Fachabteilung zur Informationstechnik. Die verbleibenden drei Lehrziele Z4 („Anpassung vornehmen“), Z6 („Fachlich mit System arbeiten“) und Z7 ("Technisch administrieren“) bilden deutlich abgeschlagen die dritte Gruppe. Darin zeigt sich zum einen, dass die technischen Ziele Z4 und Z7 nicht im Fokus der WI- und BWL-Ausbildung sind, sondern eher der hier nicht betrachteten Informatikausbildung. Zum anderen sollen sich die Studierenden beim Einsatz von ERP-Systemen nicht vorrangig auf die „Mechanik der Software" konzentrieren, sondern Kenntnisse aus unterschiedlichen betriebswirtschaftlichen Kursen (wie zum Beispiel Produktion, Logistik, Planung, Controlling, Marketing usw.) praktisch anwenden, um die Zusammenhänge besser zu verstehen (vgl. Johnson et al. 2004, S. 251f.). 


\subsection{Beziehung zwischen den Lehrzielen und den Lerneinheiten}

QFD nutzt ausgiebig sog. Priorisierungsmatrizen um zwei Informationstypen (hier Lehrziele und Lerneinheiten) miteinander in eine Beziehung zu bringen (Herzwurm u. a. 2000, S. 22-23). So ist es durch die Quantifizierung der Korrelationsstärke (i. d. R. mit den Werten 0, 1, 3 und 9), möglich, aus den bekannten Gewichtungen der Lehrziele (in Tab. 2 in den Zeilen), die Lerneinheiten (in Tab. 2 in den Spalten) zu priorisieren. Die Frage, die es gilt für jede einzelne Matrixzelle zu beantwortet, lautet: „Welche Wirkung hat die umfangreiche/anspruchsvollere Umsetzung der Lerneinheit $\mathrm{Y}$ auf die Erfüllung des Lehrziels X?" So hat z. B. die Durchführung einer detaillierten Lerneinheit zur Prozessanalyse (LE1) einen starken Einfluss (Wert 9) auf die Erreichung des Lehrziels Z1 zur Beurteilung der IstSituation im Unternehmen.

Tabelle 2: Korrelationen zwischen Lehrzielen und Lerneinheiten

\begin{tabular}{|c|c|c|c|c|c|c|c|c|c|c|c|c|c|c|c|c|c|}
\hline $\begin{array}{l}\text { Legende: } \\
\text { Die Errïlung des Kriteriums [oben] trägt } \\
9: \text { zwangsläuffignd sehr stark } \\
3: \text { merkbar aber evt. eingeschränkt } \\
1: \text { möglichererweise und nur bedingt } \\
0: \text { nur mittelbar oder ggf. gar nicht } \\
\text { zur Errillung des Lehrziels [links] bei. }\end{array}$ & 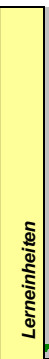 & 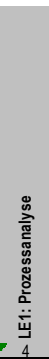 & 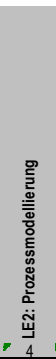 & 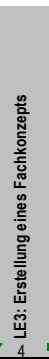 & 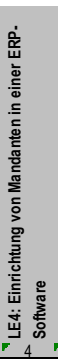 & 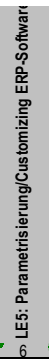 & 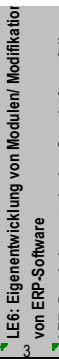 & 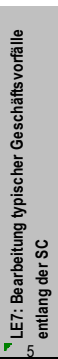 & 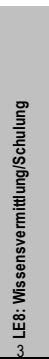 & 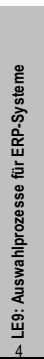 & 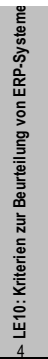 & 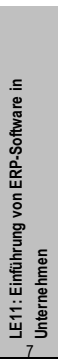 & 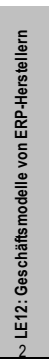 & 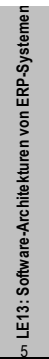 & 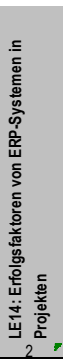 & 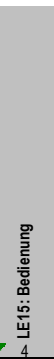 & 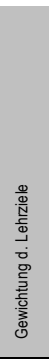 \\
\hline \multicolumn{18}{|l|}{ "Lehrziele } \\
\hline Z1: Ist-Situation beurteilen & & 9 & 3 & 1 & & & & 3 & & 1 & 3 & 3 & 1 & & 1 & & $22,3 \%$ \\
\hline Z2: Anpassungsbedarfe ident. & & 9 & 9 & 3 & 1 & 1 & & 3 & & 3 & & 3 & & 1 & & 1 & $22,1 \%$ \\
\hline Z3: Integrationsbedarfe ident. & & 9 & 3 & 9 & & 1 & & 1 & & 1 & & & & 1 & & & $18,8 \%$ \\
\hline Z4: Anpassung vornehmen & & & & & 9 & 9 & 9 & 3 & & & & 3 & & 3 & & & $6,1 \%$ \\
\hline Z5: Org. Implementierung meister & & 1 & 1 & 3 & 3 & 1 & 1 & & 3 & 3 & 3 & 9 & 9 & 1 & 9 & 1 & $16,4 \%$ \\
\hline Z6: Fachlich mit System arbeitten & & & & & & 1 & & 9 & & & & & & & & 9 & $10,9 \%$ \\
\hline Z7: Technisch administrieren & & & & & 9 & 9 & 3 & & 3 & & & 1 & & 1 & & 1 & $3,4 \%$ \\
\hline \multirow{4}{*}{$\begin{array}{l}\text { Bedeutung Lerneinheit } \\
\text { relative Bedeutung } \\
\text { Rang }\end{array}$} & $100 \%$ & & & & & & & & & & & & & & & & \\
\hline & & 5,85 & 3,39 & 3,07 & 1,57 & 1,54 & 0,82 & 2,68 & 1,58 & 1,57 & 1,57 & 3,54 & 1,70 & 0,79 & 1,70 & 1,40 & \\
\hline & & $17,9 \%$ & $10,3 \%$ & $9,4 \%$ & $4,8 \%$ & $4,7 \%$ & $2,5 \%$ & $8,2 \%$ & $4,8 \%$ & $4,8 \%$ & $4,8 \%$ & $10,8 \%$ & $5,2 \%$ & $2,4 \%$ & $5,2 \%$ & $4,3 \%$ & \multirow[t]{2}{*}{$100 \%$} \\
\hline & & 1. & 3. & 4. & 10. & 12. & 14. & 5. & 8. & 11. & 9. & 2. & 6. & 15. & 6. & 13. & \\
\hline
\end{tabular}

Die absolute Bedeutung der Lerneinheit ergibt sich aus der Spaltensumme der Multiplikationen der einzelnen Korrelationswerte in den Matrixzellen mit den zugehörigen Gewichten der Lehrziele. So errechnet sich z. B. die absolute Bedeutung von LE1 als $(9 * 22,3 \%+9 * 22,1 \%+9 * 18,8 *+1 * 16,4 \%)=5,85$. Um die relative Bedeutung der Lerneinheiten zu erhalten müssen die einzelnen absoluten Bedeutungen jeweils durch die Summe aller absoluten Bedeutungen dividiert werden. Für LE 1 bedeutet dies 5,85 dividiert durch insgesamt 32,75, d. h. 17,9\%. Damit ist die Prozessanalyse die mit Abstand aus Sicht der Erfüllung der Lehrziele wichtigste Lerneinheit. 


\subsection{Ergebnisse der Planungen des ERP-Curriculums}

Die Ergebnisse aus Kapitel 4.2 wurden bei der Planung des resultierenden ERPCurriculums berücksichtigt: Durch die begrenzten Ressourcen fand, soweit möglich, eine Fokussierung auf die wichtigsten Lerneinheiten statt, die im Sinne von Modulen zu einzelnen Lehrveranstaltungen im Bereich ERP kombiniert wurden. Die praktische Nutzung des am Betriebswirtschaftlichen Institut der Universität Stuttgart neu eingerichteten ERP-BI-Labors (www.wi.uni-stuttgart.de/erp-bi-lab) stand dabei im Mittelpunkt der Planung. Tabelle 3 gibt einen Überblick über die Abdeckung der Lerneinheiten (geordnet nach ihrer Bedeutung) durch insgesamt fünf verschiedene Lehrveranstaltungen.

Tabelle 3: Abdeckung der Lerneinheiten durch Lehrveranstaltungen

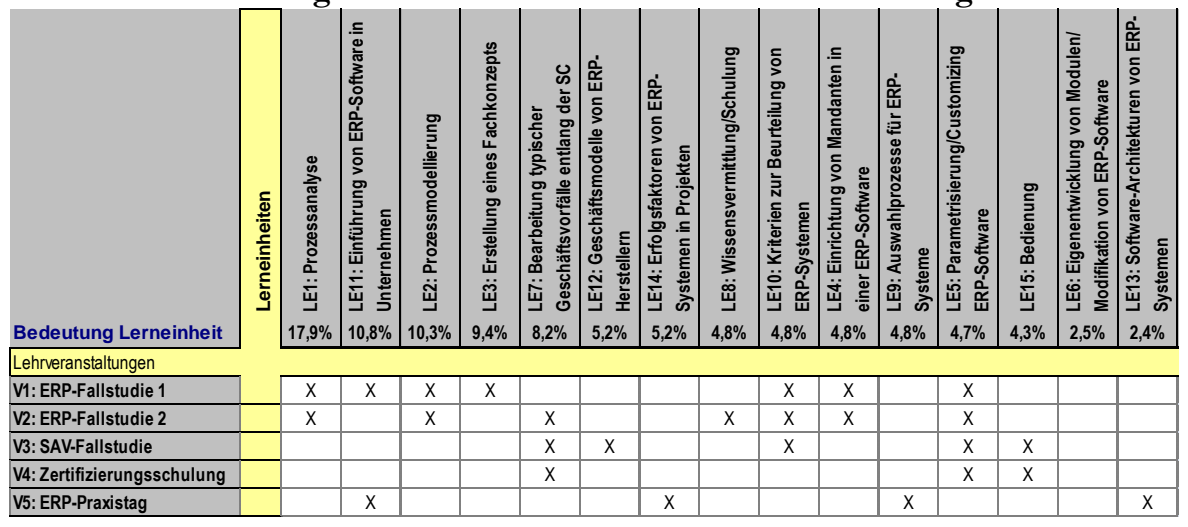

Die wichtigsten Lerneinheiten adressieren die beiden Veranstaltungen „ERPFallstudie 1“ und „ERP-Fallstudie 2“. Ziel dieser Fallstudien ist es, die Geschäftsprozessanalyse und -modellierung (LE1 und LE2) als Grundlage einer ERPEinführung kennenzulernen. Darüber hinaus sollen der einzelne Teilnehmer eine spezielle ERP-Software (Fallstudie 1: MS Dynamics NAV, Fallstudie 2: SAP R/3 bzw. SAP ECC) und deren Möglichkeiten beurteilen lernen (LE10), in dem er in der Gruppe mit anderen Studierenden eine Fallstudie selbstständig bearbeitet: In der ERP-Fallstudie 1 (V1) soll auf Basis einer Prozessanalyse ein Fachkonzept für ein Unternehmen entwickelt und modelliert (LE2+LE3) sowie durch Mandanteneinrichtung und Parametrisierung (LE4 und LE5) eine ERP-Software für ein Unternehmen implementiert werden (LE11). Bedingt durch die eingesetzte Softwarelizenz wird bei der ERP-Fallstudie 2 (V2) eine andere Herangehensweise gewählt und der Fokus auf die Bearbeitung typischer Geschäftsvorfälle (LE7) und die Vermittlung des erlangten Wissens gelegt (LE8). Die Studierenden müssen hierbei den Mandanten (LE4 und LE5) für die Aufgabenstellung anpassen und die anderen Teilnehmer zur Erledigung der Aufgabe schulen.

Die $S A V$-Fallstudie (V3, Kunstwort aus $S A P$ und $N A V$ ) beschreibt einen Prozess der kundenbezogenen Auftragsfertigung, der vergleichend in SAP ECC und in 
Microsoft Dynamics NAV durchgeführt wird. Der Vergleich ist allerdings nicht nur auf die beiden genannten Systeme beschränkt, sondern auch auf andere Systeme erweiterbar (inzwischen z. B. auch auf die Oracle E-Business Suite und Microsoft Dynamics AX). Verkürzt dargestellt wird in der Basisversion, nachdem alle notwendigen Stammdaten angelegt wurden, zuerst ein Kundenangebot erstellt, das dann in einen Kundenauftrag umgewandelt wird. Nachdem die Materialplanung durchgeführt ist, können die notwendigen Einkaufsteile beschafft und die Produkte produziert werden. Abschließend werden die Produkte geliefert und fakturiert. Über die Basisversion hinausgehende Szenarien z. B. zu der Abwicklung von Teillieferungen oder Produktionsabweichungen vertiefen dann ausgewählte Aspekte.

Die Veranstaltung richtet sich dabei an Anfänger im Umgang mit ERPSystemen. So wird jeweils eine eingehende Übung zur Bedienung aller beteiligten Systeme durchgeführt. In die Fallstudie selbst wird sowohl durch Dozenten eingeführt, es existiert aber auch eine umfangreiche schriftliche Anleitung zur Durchführung der Fallstudie. Diese ist keineswegs eine reine „Klickanleitung“ d. h. es wird vermieden, dass die Studenten nur Anweisungen aus der Fallstudie ausführen, ohne darüber nachzudenken. Es handelt sich vielmehr um einen Handlungsleitfaden, der in die Prozessschritte einführt, aber eben durchaus die eine oder andere Frage unbeantwortet lässt (z. B. in welchen Bereichen entsprechende Funktionalität zu finden ist). Auch können die Studierenden je nach Vorwissen und Anspruch weiterführende Szenarien mit offeneren Fragestellungen bearbeiten. Die selbstständige Durchführung der Fallstudie auch ohne Anleitung durch Dozenten/Tutoren ist so möglich, aber eben nicht ganz so einfach, da nicht alle Fragen in der Fallstudie vorab beantwortet werden.

Der Fokus liegt dabei auf der Bearbeitung typischer Geschäftsvorfälle entlang der Supply Chain (LE 7) und der Bedienung (LE 15), sowie auf der Parametrisierung (LE 5). Es werden auch die jeweiligen Geschäftsmodelle der Hersteller (LE 12) und anhand der Ergebnisse des Vergleichs mögliche Kriterien zur Beurteilung der ERP-Systeme vermittelt (LE 10). Als Beispiele für Unterschiede der beteiligten ERP-Systeme seien die Einfachheit der Bedienung, die Schwierigkeiten der Anpassung, aber auch die Möglichkeiten der Anpassung sowie die verschiedenen Architekturkonzepte erwähnt. Diese Vielfalt, Stärken und Schwächen mehrere Systeme, vergleichend zu erkennen und beurteilen zu können, ist ein großer Mehrwert für die Studierenden und zeugt gleichzeitig von Hersteller- und Produktunabhängigkeit. Für den genauen Ablauf der Fallstudie auch visualisiert durch entsprechende grafische Modelle sowie die begleitenden Unterlagen sei auf die Internetseiten des ERP-BI-Labors bzw. auf die Kontaktaufnahme mit den Autoren verwiesen.

Bei der SAV-Fallstudie steht die Vermittlung grundlegender Konzepte im Mittelpunkt und weniger die Schulung einzelner Produkte. Letztere können allerdings durch offizielle Zertifizierungsmöglichkeiten (V4) seitens der ERP-Softwarehersteller zusätzliche Anreize zur Teilnahme an ERP-Veranstaltungen bieten. Auch diese wurden in das ERP-Curriculum übernommen, allerdings zeigen erste Erfahrungen die Notwendigkeit einer größeren Detailtiefe in der Vermittlung der Inhalte, die 
den Umfang der Veranstaltung (auf allen Seiten) deutlich erhöhen. Gleichzeitig deckt die Zertifizierungsveranstaltung in der Breite weniger Lerneinheiten ab.

Beispielhaft für derzeit nicht durch das Curriculum abgedeckte Lerneinheiten sind die LE6 „Eigenentwicklung“ und die LE13 „Software-Architekturen von ERP-Systemen". Beide waren (und sind) allerdings Gegenstand von vertiefenden Abschluss- und Diplomarbeiten. Auch waren sie Inhalt des eingeführten ERPPraxistages (V5), bei dem Gastdozenten aus Ihrer betrieblichen Praxis über interessante Projekte berichteten.

\section{Beurteilung und Übertragbarkeit des Konzepts zur Planung eines ERP-Curriculums}

Das vorgeschlagene systematische Vorgehen stellt sicher, dass definierte Lehrziele durch den angebotenen Fächerkanon abgedeckt werden können. Die Darstellung von Lerneinheiten in einer Korrelationsmatrix erlaubt es, redundante Lehreinheiten zu identifizieren. Gleichfalls wird vermieden, dass Veranstaltungen ohne (direkten) Kundennutzen (insb. für Dozenten und Studierende) als Selbstzweck angeboten werden und knappe Ressourcen binden.

Darüber hinaus kann eine Modularisierung von Lerninhalten durch Lerneinheiten erreicht werden. Diese Einheiten können wiederum zu unterschiedlichen Lehrveranstaltungen kombiniert werden, auch um (möglichst) flexibel auf Änderungen des Umfeldes (wie z. B. Änderungen in Prüfungsordnungen oder auch Anforderungen von Arbeitgebern) reagieren zu können.

Das präsentierte Vorgehen erlaubt durch die Benutzung der inhärent flexiblen Planungsmethode QFD eine Vielzahl von Varianten. In einer Ausbaustufe könnte die Planung des Curriculums z. B. dahingehend erweitert werden, dass die Bewertung der Lehrziele durch zusätzliche Kundengruppen vorgenommen wird. Hierbei könnten Studierende aus verschiedenen Fachrichtungen (BWL/WI/Informatik) bzw. grundlegenden Bachelor- vs. vertiefenden Master-Studiengängen, Arbeitgeber oder andere Hochschulen mit unterschiedlichen Profilen infrage kommen. Je mehr unterschiedliche Sichtweisen in die Planungen einbezogen werden, desto besser kann auch möglichen Überbewertungen durch subjektive Einschätzungen entgegen gewirkt werden. Auch die Planung von unterschiedlichen Varianten des Curriculums durch Kombination von Lerneinheiten für unterschiedliche Berufsbilder (z. B. Entwickler/Anwendungsspezialist/Berater) ist denkbar.

Eine weitere Planungskomponente ist zudem die Art der Durchführung einer Veranstaltung. So ist für die in Kap. 4.4 dargestellte SAV-Fallstudie, die Variante einer Blockveranstaltung und einer themenbezogenen Veranstaltung denkbar. Von der Wahl der Lehrform kann der Nutzen der Lerneinheit entscheidend beeinflusst werden. So ist der Vergleich der Systeme in einer Blockveranstaltung dann schwieriger, wenn die Fallstudie für ein System en bloc bearbeitet wird und zwischen den Tagen der Blockveranstaltung ein relativ großer Zeitraum liegt. Wöchentliche the- 
menbezogene Veranstaltungen erlauben dagegen, die Systeme im Bezug auf einen kleineren Themenkreis zeitnah zu vergleichen, besitzen allerdings den Nachteil, dass u. U. in jeder Veranstaltung erneut zeitlicher Aufwand für die Wiederholung grundlegender Bedienungskonzepte anfällt.

Der hier präsentierte Vorschlag zur Konzeption der ERP-Lehre kann auf andere Bildungseinrichtungen und Lehrgegenstände übertragen werden. Allerdings empfiehlt es sich, Lehrziele und Lerneinheiten ggf. an die eigenen Rahmenbedingungen anzupassen. Zudem ist die Übertragbarkeit der in Kapitel 4.3 beispielhaft dargestellte vergleichende SAV-Fallstudie gegeben. Kontaktieren Sie dafür die Autoren.

\section{Literatur}

Boyle T (2007) Technical-Oriented Enterprise Resource Planning (ERP): Body of Knowledge for Information Systems Programs: Content and Implementation. Journal of Education for Business, May/June 2007: 267-274.

Cohen L (1995) Quality Function Deployment. How to Make it Work for you. Pearson Prentice Hall, Reading, Mass.

Hesseler M, Görtz M (2008) Basiswissen ERP-Systeme. W3L-Verlag, Herdecke.

Herzwurm G, Schockert S, Mellis W (2000) Joint Requirements Engineering. QFD for Rapid Customer-Focused Software Development. Vieweg, Braunschweig.

Johnson T, Lorents AC, Morgan J, Ozmun, J (2004) A Customized ERP/SAP Model for Business Curriculum Integration. Journal of Information Systems Education, 2004 (15), No. 3: 245 - 253.

Kurbel K (2009) Das Studium der Wirtschaftsinformatik. In: Kurbel K, Brenner W, Chamoni P, Frank U, Mertens P, Roithmayer (Hrsg) Studienführer Wirtschaftsinformatik 2009|2010. Gabler, Wiesbaden: 17-37.

Rang M, Hohn B, Rühl O (2008) Wirtschaftswissenschaftler: Neue Chancen auf dem Europäischen Arbeitsmarkt? Wirtschaftsstudium, Mai 2008: 283-287.

Stahlknecht P, Hasenkamp U (2005) Einführung in die Wirtschaftsinformatik. Springer, Berlin.

Strong DM, Fedorowicz J, Sager J, Stewart G., Watson E. (2006) Teaching with Enterprise Systems. Communications of AIS, 2006 (17), article 33.

Wyssusek B (2001) Systemanalyse. In: Mertens P (Haupthrsg.) Lexikon der

Wirtschaftsinformatik. Springer, Berlin: 451-453. 